\title{
A timeline for Eustoma grandiflorum seedling production based on an in vitro germination protocol
}

\author{
Md Zohurul Kadir Roni ${ }^{1^{*}}$, Md Saiful Islam ${ }^{1}$, Kazuhiko Shimasaki $^{2}$ \\ ${ }^{1}$ The United Graduate School of Agricultural Sciences, Ehime University, 3-5-7 Tarumi, Matsuyama, Ehime, 790- \\ 8566, Japan \\ ${ }^{2}$ Faculty of Agriculture and Marine Science, Kochi University, Monobe B 200, Nankoku-shi, Kochi, 783-8502, \\ Japan
}

\author{
*Corresponding author: ronisau@gmail.com
}

\begin{abstract}
Eustoma (Eustoma grandiflorum) is a high-rank ornamental cut flower, and propagation of plant material by seed is an important approach for high-volume production of Eustoma. Successful propagation using different parts of the Eustoma plant has been reported in numerous studies; however, to date there have been no studies comparing the timeline differences between in vitro and conventional germination growth methods. The objective of this study was to develop a convenient and straightforward in vitro protocol that decreases the lag time to germination and the seedling development periods. Seed germination and seedling growth was studied in Eustoma (Voyage type 2 pink) seeds using two different techniques, in vitro germination and conventional sowing in soil. Seeds sown under in vitro conditions exhibited faster (6 days) germination compared with seeds sown directly in soil (11 days). Halfstrength Murashige and Skoog medium in vitro-grown seedlings (7 weeks) were more vigorous and produced true leaves earlier compared with conventionally-grown seedlings (15 weeks). In addition, when Eustoma seeds were sown in vitro, germination could be more easily tracked and visually compared with seeds sown in soil media. Therefore, the in vitro seed germination protocol developed in this study is straightforward, reproducible, and will amenable to facilitate efficient seedling production of Eustoma for commercial applications.
\end{abstract}

Keywords: Eustoma grandiflorum; in vitro technique; seed germination and growth rate; timeline.

Abbreviations: CCFL_Cold cathode fluorescent lamps, MES-Na_2-(N-Morpholino) ethanesulfonic sodium salt, NaOCl_Sodium hypochlorite, $\mathrm{RH} \_$Relative humidity, UM_Unified mayonnaise

\section{Introduction}

Eustoma [Eustoma grandiflorum (Raf.) Shinn] is a long day plant belonging to the family Gentianaceae. It originated as a wildflower native to a region corresponding to the area between North and South America. Eustoma, also known by its common name Lisianthus, ranks high among ornamental cut flowers, and is popular for its bright, colorful petals, long post-harvest life, and the ease at which it can be shipped worldwide.

In general, Eustoma seed is tiny and germination under conventional soil-sown conditions is sensitive to a number of environmental factors including optimal light, temperature, and moisture level (Harbaugh, 1995; 2006). A photoperiod of $16 \mathrm{~h}$ light is required for Eustoma seed germination, at least until seedling emergence (Harbaugh, 1995). An appropriate photoperiod is important for regulating germination and seedling growth because of its effect on cotyledon expansion, leaf and hypocotyl development, and photosynthesis (Williams et al., 1998). In addition to specific light requirements, seed germination requires a constant temperature of $20-25^{\circ} \mathrm{C}$ (Roh et al., 1989). Emergence is delayed if temperature is not maintained carefully during the germination period (Kepczynska et al., 2006; Norsworthy and Oliveira, 2006). Moreover, high temperatures inhibit seedling production at the primary stage (Ohkawa et al., 1991). Large fluctuations between day and night temperatures also affect seedling growth and development, causing Eustoma seedlings grown in soil media to form rosettes (Ohkawa et al., 1993). Plants that form rosettes exhibit delayed flowering. In tropical regions, temperatures are higher and fluctuate more drastically compared with temperate regions, which are conditions that inhibit seed germination. Therefore, the environmental conditions in these areas would not support reliable seed germination for largescale production using conventional soil media methods without facilities to control these critical parameters.

Production and propagation of plant material by seed is an important approach for high-volume production, but it is also an important as well as being the only means for enabling the evaluation of progeny from genetic crosses for selection and development of new cultivars. Conventional, in-soil germination of Eustoma seeds for producing seedlings has been attempted, but this technique has not proved satisfactory for commercial producers and growers, due in part to the lengthy production time (Harbaugh, 1995) and including the rosette phenotype often accompanying this propagation method (Ohkawa et al., 1993; Fukuda et al., 1994).

A new era for commercialization of Eustoma has opened up through development of rapid and high-volume production techniques based on in vitro multiplication of plant material from various plant parts including the leaf and apical shoot tip (Semeniuk and Griesbach, 1987; Paek and Hahn, 2000; Esizad et al., 2012; Kaviani, 2014), flower bud or anther (Rezaee et al., 2012), internode (Semeniuk and Griesbach, 
1987; Rezaee et al., 2012), and hypocotyl. Consequently, different media recipes with varying concentrations of various components such as carbohydrates (Akbari et al., 2014), vitamin and plant growth regulators (Kaviani, 2014; Winarto et al., 2015), hormones (Paek and Hahn, 2000), and solidifying agents (Semeniuk and Griesbach, 1987; Rezaee et al., 2012) as well as different concentrations of the same media mixture have been developed to propagate Eustoma from different plant parts. However, these previous studies have not addressed the timeframe for production or resulting plant quality, aspects that are important for commercial applications. Therefore, adoption of in vitro protocols would enable manipulation of these parameters to improve germination and seedling growth rates for commercial Eustoma production in tropical regions. Seed germination protocols have already been reported and established for other plants including Aloe ferox Mill. (Bairu et al., 2009), Brasiliorchis picta (Dos Santos et al., 2016), Cymbidium aloifolium (L.) (Pradhan et al., 2014) and Annona crassiflora Mart. (Ribeiro et al., 2009) with various aims including the preservation of endangered plant species. Development of methods for the efficient germination of Eustoma seeds and growth of seedlings in vitro should similarly be achievable and applicable as an alternative method for Eustoma production. Previously, Damiano et al. (1989) reported the micropropagation and tissue culture germination of Eustoma seed, but did not present a clear protocol or a timeline relevant for commercial applications. In this paper, we present streamlined methods to enable research and production laboratories and or seed companies to rapidly adopt the technique of germinating Eustoma seeds in vitro. In summary, we (i) describe and compare the morphological characteristics of the Eustoma seedlings resulting from in vitro and conventional culture (ii) compare the production timelines obtained using in vitro and conventional culture and (iii) describe the detailed procedures to produce seedlings using an in vitro seed germination technique. This study represents the first report on a method of germination and propagation of Eustoma by seed in vitro together with a comparison of the production timelines and seedling phenotypes obtained from seeds grown in vitro versus conventional soil culture.

\section{Results and discussion}

\section{In vitro technique}

Rapid seed germination under regulated light and optimal temperature was visually apparent for seeds sown in vitro. The seedlings emerged in vitro six days after seeding (Fig. 1 and 3) and exhibited rapid growth and development to the fourth true leaf pair by seven weeks. The germination percentage, and shoot and root length were significantly greater for seeds sown in vitro compared with those sown in conventional technique; however, there was no significant difference in total fresh biomass between the two techniques (Table 1). The seedlings germinated in vitro appeared to be healthy and vigorous (Fig. 1). The rapid development of the seedlings in vitro not only reduced the duration of production but also the days to leaf pair is shorter for commercial production (Fig. 3). The in vitro technique utilized in this study employed an artificial growth medium consisting of macro- and micro-salts that provided essential elements and sucrose for controlling growth and various metabolic activities (Gibson, 1984), a combination of nutrients known to support photomixotrophic metabolism and optimal development (Coupe et al., 2006; Fila et al., 2006). Sucrose influences the maintenance of osmotic function in plant cells, directly participating in glycolytic and pentose phosphate pathways that speed up the growth and development of the seedling (Zha et al., 2007; Gago et al., 2014). Indeed, early in vitro studies reported that sucrose acts on and significantly affects seed germination, shoot emergence, leaf and root development, and seedling development (Wang and Ruan, 2014; Huh et al., 2016). Establishing protocols for seed germination and seedling growth is critical for improved seedling quality and large-scale production of crops (Subedi and Ma, 2005).

\section{Conventional technique}

In contrast to the results observed for seeds sown using the in vitro technique, germination of seeds sown in the soil-based medium (conventional seed sowing in soil) took eleven days (Fig. 3); even under the uniform, optimal conditions provided, seed germination was $84.3 \pm 0.58 \%$ (Table 1 ). However, the survival rate was higher for the plants grown using conventional seed sowing in soil compared with the in vitro technique (Table 1). Due to the seed germination to seedlings development accomplishes in soil-based medium, transfer condition that faces the minimal environmental stresses for the survival of seedlings in conventional technique compared with in vitro, however; other attributes of Eustoma seedlings were greater in vitro (Table 1). Growth and development to the fourth leaf pair for the seedlings sown in soil required at least fifteen weeks (Fig. 2 and 3). These results are in line with previous data indicating a period of 10-20 weeks for production of transplant-ready (4-5 true leaf pair stage) Eustoma seedlings from seed using conventional, soil sowing techniques (Halevy and Kofranek, 1984; Matsuo and Shirasaki, 1990; Harbaugh, 1995). According to previous reports, Eustoma seed germination in soil is finicky, requiring great skill, daily maintenance, and appropriate facilities to provide the optimal environmental conditions. Slow seed germination and lengthy production timeline have a great bearing on the overall production cost and resulting potential economic damage to seedling producer.

\section{Materials and Methods}

\section{Plant material}

The Eustoma (Voyage type 2 pink) seeds (Eustoma grandiflorum) used in this experiment were obtained from Sakata Seed Corporation, Japan. Eustoma seeds were left in a sunny place to dry and then kept in a seed storage chamber at $4^{\circ} \mathrm{C}$ for one week. Seed from any commercial seed company, nursery, or garden center can be used to demonstrate the applicability and efficacy of this technique.

\section{Media preparation for the in vitro technique}

For in vitro Eustoma seed germination, half-strength macromicro salt (Murashige and Skoog, 1962) supplemented with $30.0 \mathrm{~g} \mathrm{~L}^{-1}(3.0 \%)$ sucrose (Sigma Aldrich, USA) was used as the growing medium (Fig. 4). Phytagel (3.0 $\mathrm{g} \mathrm{L}^{-1}$; Sigma Aldrich, USA) was added and dissolved by heating the medium in a microwave oven set on high for $4 \mathrm{~min}$. The medium was mixed in an Erlenmeyer flask using a magnetic 
Table 1. Comparison of in vitro and conventional technique on growth and development of Eustoma.

\begin{tabular}{lccccc}
\hline Culture technique & $\begin{array}{c}\text { Germination } \\
\text { percentage }(\%)\end{array}$ & $\begin{array}{c}\text { Shoot length } \\
(\mathrm{mm})\end{array}$ & Root length $(\mathrm{mm})$ & $\begin{array}{c}\text { Total fresh biomass } \\
(\mathrm{g})\end{array}$ & $\begin{array}{c}\text { Survival rate } \\
(\%)\end{array}$ \\
\hline in vitro & $96.09 \pm 0.40$ & $56.6 \pm 0.82$ & $72.2 \pm 0.62$ & $1.1 \pm 28.2$ & $82.3 \pm 0.93$ \\
Conventional & $84.3 \pm 0.58$ & $48.7 \pm 0.94$ & $63.4 \pm 0.80$ & $0.8 \pm 22.7$ & $92.3 \pm 0.61$ \\
\hline
\end{tabular}

Traits are mean \pm SE $(n=10)$ and significantly different $(p<0.05)$ using Tukey's HSD test.
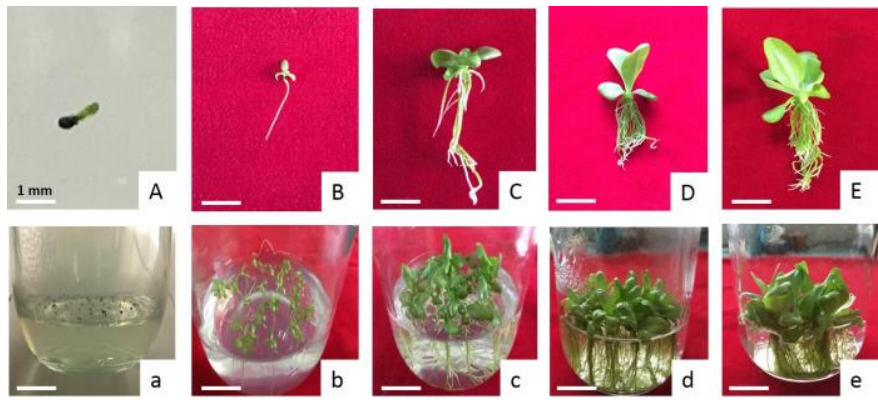

Fig 1. Development of Eustoma (Eustoma grandiflorum) seedlings from germination to seedling stage in vitro. A and a, seed emergence and germination (6 days); B and b, first true leaf pair stage (1 week); C and c, second true leaf pair stage (3 weeks); D and $\mathrm{d}$, third true leaf pair stage (5 weeks); E and e, fourth true leaf pair stage (7 weeks). Scale bar $=2 \mathrm{~cm}$.
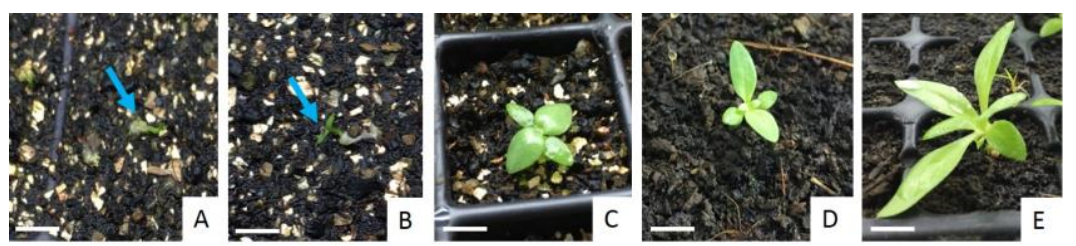

Fig 2. Seedling development of Eustoma (Eustoma grandiflorum) plants seeded in conventional, soil medium. A, seed germination (11 days); B, first true leaf pair stage ( 3 weeks); C, second true leaf pair stage (5 weeks); D, third true leaf pair stage ( 8 weeks) and E; fourth true leaf pair stage (15 weeks). Scale bar $=2 \mathrm{~cm}$.

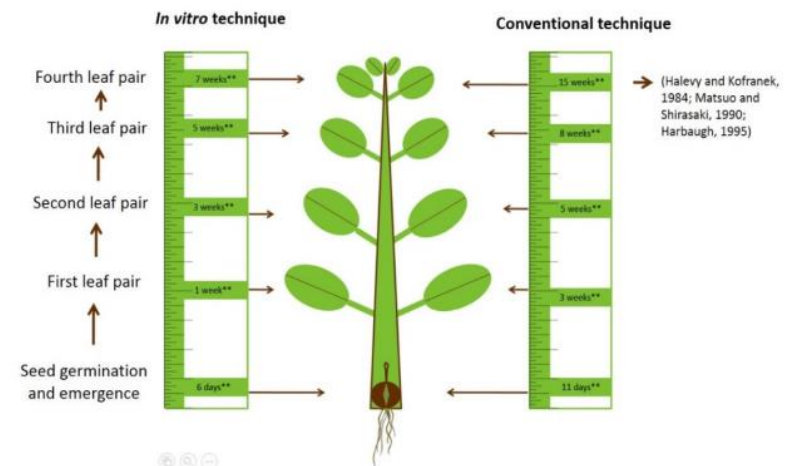

Fig 3. A timeline for Eustoma (Eustoma grandiflorum) seed germination through various stages of seedling development. A flow chart outlining the timeline (in days and weeks) for the various seedling developmental stages of plants obtained from seeds sown in vitro versus by plants obtained from seeds sown by the conventional technique (in soil). (Numbers in each column represent the days to germination and weeks to respective number true leaf pair. ** showing significant difference with Tukeys HSD test at $p<0.05$. Traits are mean \pm SE and $n=10$ )

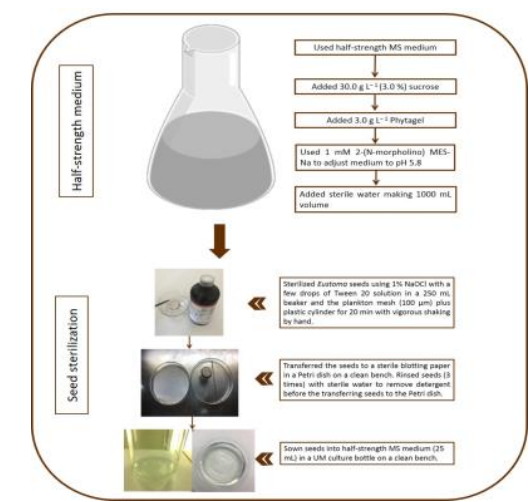

Fig 4. Scheme for the preparation of a $1000 \mathrm{ml}$ of half-strength MS medium and the seed sterilization technique for Eustoma seed germination and growth in vitro. 
stir bar and a stir plate (AS ONE, Japan) set at $600 \mathrm{rpm} \mathrm{min}^{-1}$ for $3 \mathrm{~min}$. The medium was adjusted to $\mathrm{pH} 5.8$ with $1 \mathrm{mM} 2-$ (N-morpholino) ethanesulfonic sodium salt (MES-Na) (Sigma Aldrich, USA). The culture medium was sterilized by autoclaving at $121^{\circ} \mathrm{C}$ and $1.5 \mathrm{kgf} \mathrm{cm}$ for $15 \mathrm{~min}$ (Tomy BS305; Tomy Seiko Co., Ltd., Japan). Following autoclaving, the medium was distributed aseptically in $25 \mathrm{~mL}$ aliquots to sterile UM (Unified mayonnaise clear glass culture bottle with plastic cap) bottles (AS ONE, Japan) under laminar air flow (Bio Clean Bench; Sanyo Electric Co., Ltd., Japan) and then allowed to solidify for $20 \mathrm{~min}$, after which it was stored until use.

\section{Seed sterilization for the in vitro technique}

The seeds were disinfected in a laminar flow chamber. Seeds $(0.15 \mathrm{~g})$ were placed in a $3 \mathrm{~cm}$ diameter plastic sieve cylinder fitted with plankton mesh $(100 \mu \mathrm{m}$ mesh and $5 \times 5 \mathrm{~cm}$ square net) and placed in a $250 \mathrm{~mL}$ beaker. Sterilization solution was prepared by adding $50 \mathrm{~mL}$ of $1 \% \mathrm{NaOCl}$ (Sigma Aldrich, USA) along with $500 \mathrm{~mL}$ autoclaved sterile water in a separate, $500 \mathrm{~mL}$ beaker. A few drops of Tween 20 (Sigma Aldrich, USA) solution were added with the sterilization solution to wash the seeds in the $250 \mathrm{~mL}$ beaker. The seeds held in the mesh were then surface sterilized by adding sterilization solution followed by soaking for $20 \mathrm{~min}$ (Sauer and Burroughs, 1986). The seeds were then washed three times with sterile water to remove the detergent. Vigorous shaking by hand was important to ensure thorough washing and removal of seeds clinging to the plastic sieve cylinder. The seeds were subsequently rinsed three times with sterile water (about $50 \mathrm{~mL}$ each time) in a beaker to remove the seeds clinging to the plastic sieve cylinder. After rinsing, the seeds were transferred to sterile blotting paper in a Petri dish in the flow bench. A sterile L-shaped hockey stick $(130 \mathrm{~mm} \mathrm{~L}$ $\times 50 \mathrm{~mm} \mathrm{~W}$ (bottom) $\times 4 \mathrm{~mm}$ diameter) (Sigma Aldrich, USA) was used to distribute the seeds on the surface of the growth medium contained in the UM culture bottle. There were 20-25 seeds per UM culture bottle, evenly distributed on the surface of the germination medium (Fig. 4). Higher seed densities reduce germination and development (Rasmussen and Johansen, 1989). Following seed distribution, all UM culture bottles were sealed with two layers of parafilm. All cultures were kept in a growth chamber at $23 \pm 2^{\circ} \mathrm{C}$ under a $16 \mathrm{~h}$ light photoperiod with a light intensity of $98 \mu \mathrm{mol} \mathrm{m} \mathrm{m}^{-2} \mathrm{~s}^{-1}$ (LA-105; NK-System, Japan) provided by cool white fluorescent lamps (Tubular fluorescent lamps; Toshiba Lighting \& Technology Taiwan Co., Ltd., Japan Light Analyzer). Cultures were examined every other day and contaminated cultures discarded if found.

\section{Seed sowing in soil for the conventional culture}

To understand the efficacy and merits of seed germination in vitro, seed germination was also tested using conventional methods by direct sowing in soil (Tanekura No. 42; Sumirin Agricultural Industry Co., Ltd., Japan). The germination experiment was conducted in a cold cathode fluorescent lamp (CCFL) growth chamber (Bio Multi Incubator; NK-System, Japan) under a $16 \mathrm{~h}$ photoperiod with $60-70 \%$ relative humidity (RH). Seed was sown on the surface (not buried) of Tanekura No. 42 soil contained in cells of plastic cell trays (Cell trays with 144 to 228 cells, each cell $2.5 \times 2.5 \mathrm{~cm}$ ); 50 cells, each planted with one seed each were used for germination and seedling growth tests. The plastic, seeded cell tray was subsequently covered with a polyethylene sheet to minimize moisture loss. A water contained plastic bowl
$(2.0 \mathrm{~mL})$ was placed under the plastic cell tray to maintain moisture. RH was found to be $70-75 \%$. The seeds were considered as germinated when emergence (hypocotyl) was observed. The germination period was 9-11 days. The rate of germination of the seeds sown in the soil was estimated using a modified Timson's index of germination velocity $=\Sigma \mathrm{G} / \mathrm{t}$, where $G$ is the percentage of seed germination at one-day intervals and $t$ is the total germination period (Khan and Ungar, 1984). A high mean value represents a high rate of germination.

\section{Statistical analysis}

For each treatment, there were ten replications and the results were expressed as mean \pm standard error (SE). Data from after the in vitro technique at 7 weeks and conventional sowing in soil at 15 weeks were analyzed using one-way ANOVA and significant differences were calculated after applying Tukey's HSD test at $p<0.05$ using KaleidaGraph4.5.0 (Synergy Software, USA).

\section{Conclusion}

This protocol paper incorporates practical, technical, and horticultural aspects relevant to the efficient germination of Eustoma seeds and growth of resulting seedlings in vitro. We present a simple and efficient in vitro technique for Eustoma seed germination, which effectively shortens the seed germination and seedling development periods. Short plant production timelines are crucial for reducing costs and commercial viability. In addition, the technique and results for seed germination and seedling growth of Eustoma described in detail in this study supports the importance of these parameters for improved seedling quality and largescale production. This technique can be applied to enable eustoma seedling production in tropical regions.

\section{Acknowledgements}

The author thanks to Laboratory of Floriculture and Vegetable Science, Kochi University, Japan for research and also Japanese Government for providing a (Monbukagakusho: MEXT) Scholarship to MZK Roni. The authors also gratefully acknowledge Dr. Jon Y. Suzuki for comments on an earlier version of the manuscript.

\section{References}

Akbari H, Pajooheshgar R, Karimi N (2014) Evaluating the micropropagation of lisianthus (Eustoma grandiflora L.) as an important ornamental plant. Indian J Fund Appl Life Sci. 4: 596-602.

Bairu MW, Kulkarni MG, Street RA, Mulaudzi RB, Van Staden J (2009) Studies on seed germination, seedling growth, and in vitro shoot induction of Aloe ferox Mill., a commercially important species. HortSci. 44(3): 751-756.

Coupe SA, Palmer BG, Lake, JA, Overy SA, Oxborough K et al. (2006) Systemic signalling of environmental cues in arabidopsis leaves. J Exp Bot. 57: 329-341.

Damiano C, Ruffoni B, Curir P, Esposito P, Massabò F (1989) Tissue culture and micropropagation of Lisianthus russellianus Hook. Acta Hortic. 251: 141-146.

Dos Santos SA, de Camargo Smidt E, Padial AA, Ribas LLF (2016) Asymbiotic seed germination and in vitro propagation of Brasiliorchis picta. African J Biotechnol. 15(6): 134-144. 
Esizad SG, Kaviani B, Tarang A, Zanjani SB (2012) Micropropagation of lisianthus (Eustoma grandiflorum), an ornamental plant. POJ. 5(3): 314-319.

Fila G, Badeck F, Meyer S, Cerovic Z, Ghashghaie J (2006) Relationships between leaf conductance to $\mathrm{CO}_{2}$ diffusion and photosynthesis in micropropagated grapevine plants, before and after ex vitro acclimatization. J Exp Bot. 57: 2687-2695.

Fukuda Y, Ohkawa K, Kanematsu K, Korenga M (1994) Classification of Eustoma grandiflorum (Raf.) Shinn. cultivars on rosette characteristics based on the bolting ratios after a high temperature treatment. J Jpn Soc Hortic Sci. 62(4): 845-856

Gago J, Martínez-Núñez L, Landín M, Flexas J, Gallego PP (2014) Modeling the effects of light and sucrose on in vitro propagated plants: A multiscale system analysis using artificial intelligence technology. PLOS ONE. 9(1): 0085989.

Gibson SI (1984) Plant sugar-response pathways. Part of a complex regulatory web. Plant Physiol. 124(4): 15321539.

Halevy AH, Kofranek AM (1984) Evaluation of lisianthus as a new flower crop. HortScience. 19: 845-847.

Harbaugh B (2006) Lisianthus. Flower breeding and genetics, pp. 644-663.

Harbaugh BK (1995) Flowering of Eustoma grandiflorum (Raf.) Shinn. cultivars influenced by photoperiod and temperature. HortSci. 30: 1375-1377.

Huh YS, Lee JK, Nam SY, Hong EY, Paek KY, Son SW (2016) Effects of altering medium strength and sucrose concentration on in vitro germination and seedling growth of Cypripedium macranthos Sw. J Plant Biotechnol. 43(1): 132-137.

Kaviani B (2014) Micropropagation of ten weeks (Matthiola incana) and lisianthus (Eustoma grandiflorum) (two ornamental plants) by using kinetin (KIN), naphthalene acetic acid (NAA) and 2, 4-dichlorophenoxyacetic acid (2, 4-D). Acta Sci Pol-Hortoru. 13(1): 141-154.

Kepczynska E, Piekna-Grochala J, Kepczynski J (2006) Hormonal regulation of tomato seed germination at a supraoptimal temperature. J Acta Physiol Plant. 28: 225231.

Khan MA, Ungar IA (1984) The effect of salinity and temperature on the germination of polymorphic seeds and growth of Atriplex triangularis Willd. Am J Bot. 71: 481489.

Matsuo T, Shirasaki T (1990) Effect of rate of fertilization on the growth and nutrient uptake of Eustoma grandiflorum. J Jpn Soc Hortic Sci. 59(1): 584-585.

Murashige T, Skoog F (1962) A revised medium for a rapid growth and bioassays with tobacco tissues cultures. Physiol Plantarum. 15(3): 473-479.

Norsworthy JK, Oliveira MJ (2006) Sicklepod (Senna obtusifolia) germination and emergence as affected by environmental factors and seeding depth. Weed Sci. 54: 903-909.
Ohkawa K, Kano A, Kanematsu K, Korenaga M (1991) Effects of air temperature and time on rosette formation in seedlings of Eustoma grandiflorum (Raf.) Shinn. Sci Hortic. 48(1): 171-176.

Ohkawa K, Korenaga M, Yoshizumi T (1993) Influence of temperature prior to seed ripening and at germination on rosette formation and bolting of Eustoma grandiflorum. Sci Hortic. 53(3): 225-230.

Paek KY, Hahn EJ (2000) Cytokinins, auxins and activated charcoal affect organogenesis and anatomical characteristics of shoot-tip cultures of lisianthus [Eustoma grandiflorum (Raf.) Shinn]. In Vitro Cell Dev Biol-Plant. 36(2): 128-132.

Pradhan S, Tiruwa B, Subedee BR, Pant B (2014) In vitro germination and propagation of a threatened medicinal orchid, Cymbidium aloifolium (L.) Sw. through artificial seed. Asian Pac J Trop Biomed. 4(12): 971-976.

Rasmussen H, Johansen B (1989) Density-dependent interactions between seedlings of Dactylorhiza majalis (Orchidaceae) in symbiotic in vitro culture. Physiol Plantarum. 77(4): 473-478.

Rezaee F, Ghanati F, Boroujeni LY (2012) Micropropagation of lisianthus (Eustoma grandiflorum L.) from different explants to flowering onset. Iran J Plant Physiol. 3(1): 583-587.

Ribeiro MDNO, Pasqual M, Villa F, Pio LAS, Hilhorst HWM (2009) In vitro seed germination and seedling development of Annona crassiflora Mart. Sci Agric. 66(3): 410-413.

Roh MS, Halevy AH, Harold EW (1989) Eustoma grandiflorum. In: AH Halevy (Ed.), Handbook of Flowering, Vol. VI. CRC Press, Boca Raton, Fla, pp. 322327.

Sauer DB, Burroughs R (1986) Disinfection of seed surfaces with sodium hypochlorite. Phytopathology. 76(7): 745749.

Semeniuk P, Griesbach RJ (1987) In vitro propagation of prairie gentian. Plant Cell Tiss Org. 8(3): 249-253.

Subedi KD, Ma BL (2005) Seed priming does not improve corn yield in a humid temperate environment. Agron J. 97(1): 211-218.

Wang L, Ruan YL (2013) Regulation of cell division and expansion by sugar and auxin signaling. Front Plant Sci. 4: 163.

Williams J, Phillips A, Gaskin P, Hedden P (1998) Function and substrate specificity of the gibberellin 3b-hydroxylase encoded by the arabidopsis GA4 gene. Plant Physiol. 117(2): 559-563.

Winarto B, Rachmawati F, Setyawati AS, da Silva JAT (2015) Leaf-derived organogenesis in vitro for mass propagation of lisianthus (Eustoma grandiflorum (Raf.) Shinn). Emir J Food Agric. 27(6): 495.

Zha XQ, Luo JP, Jiang ST, Wang JH (2007) Enhancement of polysaccharide production in suspension cultures of protocormlike bodies from Dendrobium huoshanense by optimization of medium compositions and feeding of sucrose. Process Biochem. 42: 344-351. 